\title{
THE CLASSIFICATION OF MAPS OF SURFACES
}

\author{
BY DAVID GABAI ${ }^{1}$ AND WILLIAM H. KAZEZ ${ }^{2}$
}

In this note we discuss the topology of maps of positive degree between closed orientable surfaces. Two maps $f, g: M \rightarrow N$ are said to be equivalent if there exist homeomorphisms $h: M \rightarrow M$ and $k: N \rightarrow N$ such that $k \circ f=g \circ h$ (or $k \circ f \simeq g \circ h$ in the homotopy category). If $k$ is homotopic to $\operatorname{id}_{N}$ we say $f$ and $g$ are strongly equivalent. The notion of equivalence is analogous to a change of basis in domain and range in linear algebra.

Surface maps of special interest are branched coverings, i.e., $f: M \rightarrow N$ is a branched covering if there exists a finite set of points $B \subset N$ such that $f \mid M-f^{-1}(B)$ is a covering map. An arbitrary branched covering may be approximated by a generic branched covering, i.e., one in which each point of $N$ has degree $(f)$ or degree $(f)-1$ preimages.

One of the first people to study branched coverings was Riemann, who proved in his thesis (1851) that Riemann surfaces occur as conformal branched coverings of $S^{2}$. In 1871 and 1873 the classical function theorists Lüroth and Clebsch succeeded in showing that generic branched coverings of $S^{2}$ are classified up to (strong) equivalence by their degree. The classification problem for general range $N$ was reduced by Hurwitz in 1891 to the algebraiccombinatorial study of representations of $\pi_{1}(N-B)$ into $\Sigma_{d}$, the symmetric group on $d$ letters where $d=$ degree of the branched covering.

In 1928 Reidmeister showed that there is a 1-1 correspondence betweeen subgroups of $\pi_{1}(N)$ and covering spaces of $N$. This allows a generic branched covering $\phi: M \rightarrow N$ to be factored uniquely as a primitive (surjective on $\pi_{1}$ ) generic branched covering $\tilde{\phi}: M \rightarrow \tilde{N}$ followed by an unbranched covering map $p: \tilde{N} \rightarrow N$ corresponding to the image of $\phi$ on $\pi_{1}$.

Primitive generic branched coverings were shown to be classified by their degree by Hamilton in 1966 for arbitrary $N$ provided that $b \geq 2 d$, where $b$ is the number of branch points and $d$ is the degree. This was improved by Berstein and Edmonds in 1979 and 1984 to $b>d / 2$ and arbitrary $N$, or with no restriction on $b$ to $N=S^{1} \times S^{1}$. More importantly, Berstein and Edmonds stressed that primitive generic branched coverings should be classified up to equivalence by their degree and they conjectured a suggestive normal form.

Recently we have shown that primitive generic branched coverings are actually classified up to strong equivalence by their degree, and consequently we prove the following theorem.

Received by the editors September 30, 1985.

1980 Mathematics Subject Classification (1985 Revision). Primary 57M12; Secondary $14 \mathrm{H} 30$.

${ }^{1}$ Research supported in part by NSF grant DMS84-03645.

${ }^{2}$ Research supported in part by NSF grant DMS84-04535.

(C) 1986 American Mathematical Society $0273-0979 / 86 \$ 1.00+\$ .25$ per page 
THEOREM 1. Two generic branched coverings $\phi, \psi: M \rightarrow N$ of closed orientable surfaces are strongly equivalent if and only if degree $(\phi)=\operatorname{degree}(\psi)$ and $\phi_{\#} \pi_{1}(M)=\psi_{\#} \pi_{1}(M)$.

As a corollary we deduce the homotopy classification of surface maps.

COROLlaRY 2. If $f, g: M \rightarrow N$ have positive degree then $f$ and $g$ are strongly equivalent in the pointed homotopy category if and only if degree $(f)=$ $\operatorname{degree}(g)$ and $f_{\#} \pi_{1}(M)=g_{\#} \pi_{1}(M)$.

ProOF. Diagram 1 summarizes results of Nielsen 1927 (column 1) and Edmonds 1978 (column 2). The entry in each box is a map which necessarily exists in a given homotopy class of maps from $M$ to $N$. (A pinch is a map which contracts a subsurface of $M$ with connected boundary to a point.)

\begin{tabular}{c|c|c}
$\simeq$ & injective on $\pi_{1}$ & primitive \\
\hline degree 1 & homeomorphism & pinch \\
\hline degree $>1$ & covering map & $\begin{array}{c}\text { generic } \\
\text { branched } \\
\text { covering }\end{array}$
\end{tabular}

DIAGRAM 1

Since $f$ and $g$ may be written as primitive maps followed by the same covering map, the corollary follows directly from Theorem 1 .

Since surfaces are $K(\pi, 1)$ 's the last corollary gives a classification of homomorphisms of surface groups.

COROLlaRY 3. If $f, g: G \rightarrow H$ are homomorphisms of surface groups of equal topological degree greater than zero such that $f(G)=g(G) \subset H$ then there exists an isomorphism $h: G \rightarrow G$ such that $f=g \circ h$.

IDEA OF THE PROOF OF THE THEOREM. The proof of the theorem starts with the idea introduced by Gabai in his proof of the simple loop conjecture (1985) of factoring a map $\phi: M \rightarrow N$ as a branched immersion $s: M \rightarrow N \times I$ followed by a projection $\pi: N \times I \rightarrow N$. In this way the branched covering is "identified" with the space $s(M) \subset N \times I$.

As Figure 1 shows, slight changes in $s$ may result in quite different sets of double curves in $s(M)$. By applying various such topological manipulations to $s$ we eventually arrive at a branched immersion whose double curves when projected onto $N$ are as in Figure 2.

Figure 2 shows the normal form for a primitive generic branched covering of degree $d$. The domain may be visualized as an immersed subset of $N \times I$ by first embedding $d$ parallel copies of $N$ in $N \times I$, then cutting the $i$ and $i+1$ copies along each curve labelled $i$, and finally interchanging sheets and gluing. See Figure 3. 

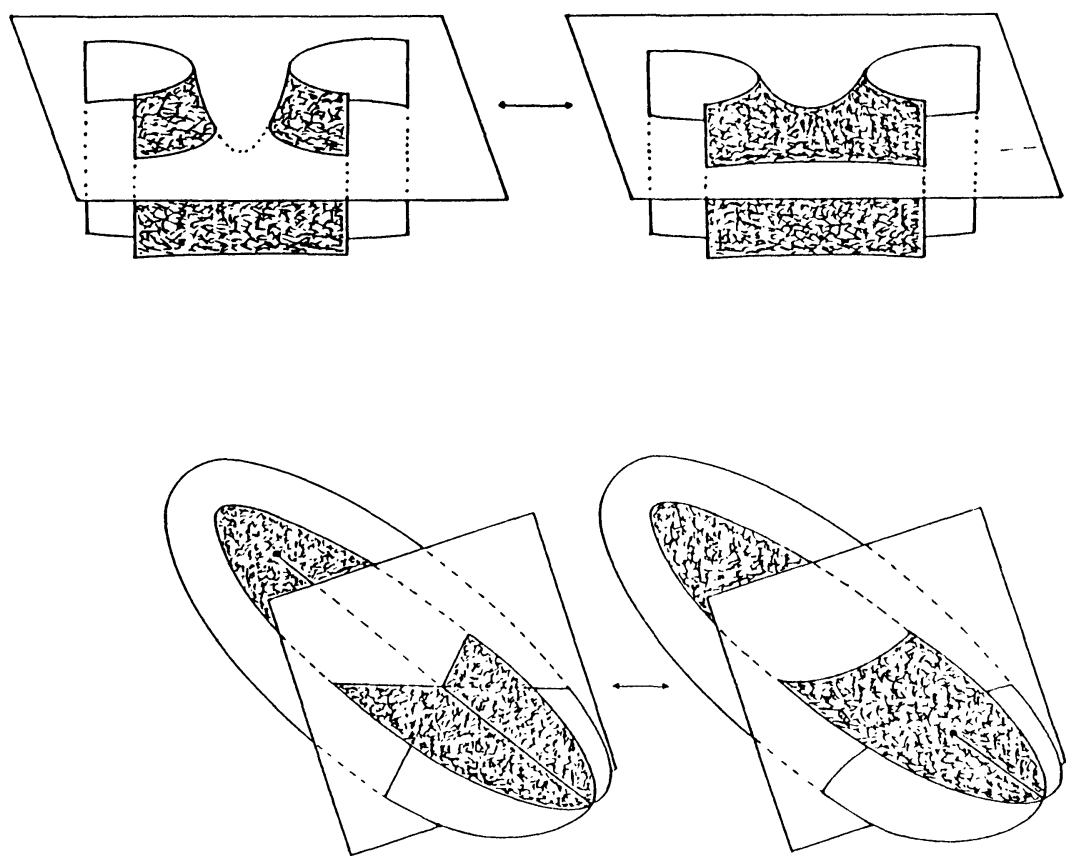

FIGURE 1
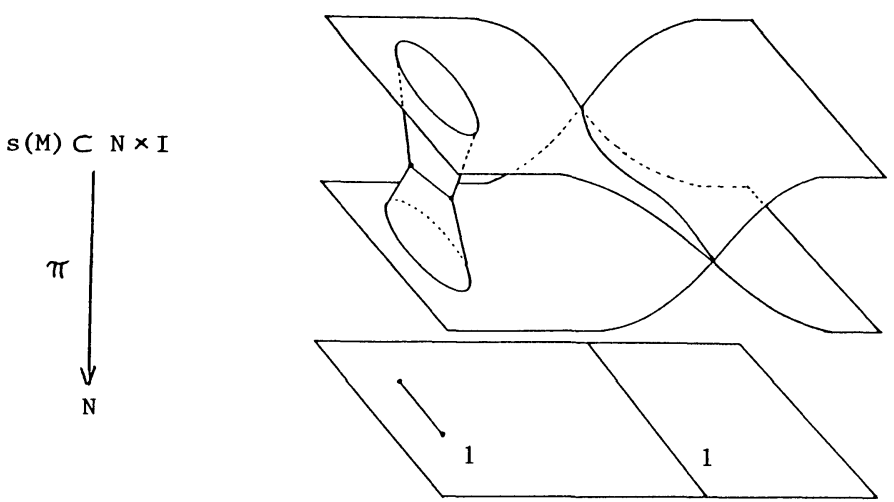

FIGURE 2 

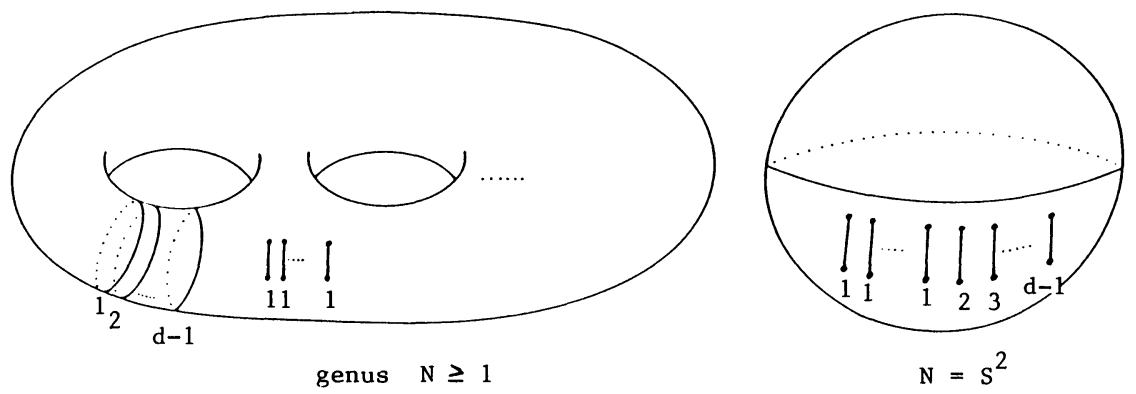

FIGURE 3

\section{REFERENCES}

I. Berstein and A. Edmonds, On the construction of branched coverings of lowdimensional manifolds, Trans. Amer. Math. Soc. 247 (1979), 87-124.

On the classification of generic branched coverings of surfaces, Illinois $\mathrm{J}$. Math. 28 (1984), 64-82.

A. Clebsch, Zur Theorie der Riemann'schen Flächen, Math. Ann. 6 (1873), 216-230.

A. Edmonds, Deformation of maps to branched coverings in dimension two, Ann. of Math. (2) 110 (1979), 113-125.

D. Gabai, The simple loop conjecture, J. Differential Geom. 21 (1985), 143-149.

R. Hamilton, Variation of structure on Riemann surfaces, Princeton University Thesis, 1966.

A. Hurwitz, Über Riemann'sche Flächen mit gegebenen Verzweigungs-punkten, Math. Ann. 39 (1891), 1-60.

J. Luroth, Note über Verzweigungsschnitte und Querschnitte in einer Riemann's chen Fläche, Math. Ann. 4 (1871), 181-184.

J. Nielsen, Untersuchungen zur Topologie der geschlossenen zweiseitigen Flächen, Acta Math. 50 (1927), 189-358.

K. Reidemeister, Fundamentalgruppe und Überlagerungsräume, Nachrichten, Göttingen 1928, pp. 69-76.

G. F. B. Riemann, Grundlagen für eine allgemeine Theorie der Funktionen einer veränderlichen complexen Grösse, Gesammelte Mathematicshe Werke, Dover, New York, 1953 , pp. 3-43.

Mathematical Sciences Research institute, 1000 Centennial Drive, BERKELEY, CALIFORNIA 94720 (Current address of D. Gabai)

Department of Mathematics, University of Pennsylvania, PhiladelPhia, PENNSYlVANIA 19104 